\title{
Clinical importance of jaw elevation during conscious sedation in pediatric patients
}

\author{
Gaurav Verma (M.D.S.) \\ Senior Lecturer, Department of Oral and Maxillofacial Surgery, Himachal Institute of Dental Sciences, \\ Paonta Sahib, Himachal Pradesh, India \\ *Corresponding Author E-mail: gauravjournals107@gmail.com
}

\begin{abstract}
Pediatric patients differ from adult in behavioral aspects. The reason for this is that, they are in developing stage and may not have the ability to understand the things similar to adults. Therefore, to perform surgical procedures in pediatric patients some form of behavioral modification techniques along with pharmacological measures may be required. Conscious sedation is one of the commonly employed techniques in this regard. Respiratory depression or arrest is a life threatening complication which may occur during conscious sedation. The purpose of this note is to draw attention of anesthesiologist toward importance of jaw elevation during conscious sedation in pediatric patients and to initiate future studies to evaluate the efficacy of newer jaw elevation device in achieving this goal.
\end{abstract}

Keywords: Conscious; Unconscious; Sedation; Respiratory Arrest; Cardiac Arrest; Jaw Elevation.

\section{Introduction}

A patient is considered conscious if the ability to rationally respond to verbal or physical stimulus is present, patient is able to maintain the airway and protective reflexes are intact (Bennett 1990, p. 268).

Conscious sedation may be defined as the establishment of a state of relaxation and freedom from fear anxiety in the conscious patient with the help of drugs (Bennett 1990, p. 269). In other words, conscious sedation can be defined as medically controlled state of depressed consciousness in which all protective reflexes are intact, patient is able to maintain patency of airway and can respond appropriately to verbal command or physical stimulation (McDonald et al. 2004, p. 287)

Deep sedation represents a deeper state of depressed consciousness from which patient cannot be easily aroused. This state may be achieved by administration of the same drugs as for conscious sedation when used at higher doses. The additional risks associated with deep sedation are partial or complete loss of protective reflexes, inability to maintain patency of airway independently and respond to verbal command or physical stimuli (McDonald et al. 2004, p. 287).

\section{Why Pediatric Patients are More Prone for Respiratory Obstruction and Respiratory Arrest}

There are anatomical and physiological differences between pediatric and adult patients. Apart from difference in age, size and weight, various tissue systems are less mature. In pediatric patients the alveolar system is less mature and oxygen demand is much higher. Physiologically rate of respiration is higher in these patients to meet the oxygen demand. Drugs which depress the respiratory system must be administered with caution in pediatric patients (McDonald et al. 2004, p. 288).

The infant and children are at increased risk for development of respiratory obstruction and respiratory arrest as compared to adults. In comparison to adults, the airway of infants and children has relatively larger adenoids and tonsils; proportionately large tongue in relation to the size of oropharynx; small sized nasal passage; and a smaller, more compliant trachea that is easily collapsed in the presence of increased airway resistance (Malamed 2007 , p. 522). Patients in which tonsillar tissues occupy $>50 \%$ of the pharyngeal space are at higher risk of respiratory obstruction (McDonald et al. 2004, p. 288).

Additional factor which predisposes the infant and smaller children to increased risk of respiratory and cardiac arrest is higher basal metabolic rate (BMR). As compared to adults, the oxygen consumption is about twice in children. Smaller functional residual lung capacity, limited oxygen reserve, physiological collapse of smaller airway in case of airway resistance may result in rapid development of hypoxia secondary to airway obstruction (Quan \& Franklin 2000, p. 43).

\section{Catastrophic Effects of Respiratory Ob- struction}

During treatment of pediatric patients, respiratory depression or arrest most commonly occurs secondary to use of central nervous system depressant drugs (CNS depressants). These drugs are used for the purpose of conscious sedation, deep sedation, or general anesthesia in conjunction with local anesthetic administration (Goodson 1983, p. 239).

If respiratory depression gets undiagnosed or managed improperly, then it can lead to catastrophic complications like cardiac arrest and death, or survival with severe or permanent neurological deficit (Malamed 2007, p. 525). 
Cardiac arrest is rare in children and when it occurs, it is predominantly due to non-cardiac causes [International consensus on cardiopulmonary resuscitation (CPR) and emergency cardiovascular care (ECC) science with treatment recommendations 2005). In children cardiac arrest typically represents the terminal event of prolonged respiratory failure, arrest or airway management problem (Kuisma et al. 1995 , p. 141, Sirbaugh et al. 1999, p. 174). Inadequate airway management and monitoring during conscious sedation is common in case of pediatric cardiac arrest (Goodson 1983, p. 239).

Pediatric patients and adults differ in cardiovascular system (CVS) parameters as well. The heart rate is higher and blood pressure is lower than in adults. Children are much more susceptible to develop bradycardia, decreased cardiac output and hypotension. Compensatory mechanisms to regulate adequate blood pressure in the presence of bradycardia are not well developed in children. Therefore, a decrease in heart rate leads to corresponding fall in blood pressure and tissue oxygenation. Great care must be taken while giving drugs which can depress CVS (McDonald 2004, p. 288)

\section{Why There is Need for Jaw Elevation Dur- ing Conscious Sedation}

Children have large occiput which leads to flexion of the neck when placed on a flat surface. Additionally pediatric patients have relatively larger tongue as compared to adults, and the epiglottis is floppy and U-shaped. Decreased tone of the pharyngeal muscles and tongue leads to increased airway resistance or obstruction due to approximation of posterior wall of pharynx and tongue (as tongue fall back) (Schmitt 2011, p. 21)

\section{Rationale for Jaw Elevation}

Chin lift, head tilt-chin lift technique and jaw thrust techniques are the airway maneuvers to maintain the patency of the airway (Malamed 2007, p. 125). Research conducted during past 20 years, established that conventional chin lift-head tilt and jaw thrust provides the most consistently reliable airway (Malamed 2007, p. 126). These maneuvers are based on the principle to stretch the tissue between the larynx and mandible, elevate the base of the tongue away from the posterior pharyngeal wall. These techniques relieve anatomic airway obstruction caused by soft tissues in $80 \%$ of the unconscious patients (Malamed 2007, p. 126).

\section{Discussion}

Safety of conscious sedation can be predicted upon a number of factors including drug titration and continuous patient monitoring (Malamed 2007, p. 522). Titration is defined as administration of drug in small incremental doses to the patient until the desired clinical effect is achieved. Titration of drug during administration is a safety factor during conscious sedation. However, only those drugs which are administered by intravenous and inhalation route have this ability. On the other hand drugs administered by oral, intramuscular, or intranasal routes have significantly variable onset of action and cannot be titrated. The pediatric dose of these routes is calculated by favorable response of $70 \%$ of patients to a given dose. However, $15 \%$ of the patients may not achieve the desired level of sedation (called as hypo-responders) and the remaining $15 \%$ of the patients May exhibits exaggerated responses (called as hyper-responders) (Malamed 2007, p. 522).

Although, oral route of drug administration is most convenient, it is least reliable. Drug doses are usually "educated guesses" based on age or body size. Also, the onset of drug action and duration are least predictable by this route. Intramuscular and subcutaneous routes may be employed for conscious sedation, but they lack the basic advantage of intravenous route (titration) (Bennett 1990, p 274).
Therefore, the possibility of undesirable CNS depression cannot be ignored with use CNS depressants via these routes.

\section{Conclusion}

From review of literature it can be concluded that, there is possibility that patient may shift from a planned state of conscious sedation to unplanned state of deep sedation or unconsciousness. This probability is further enhanced if CNS depressant drugs were administered via a route in which titration is not feasible. Anatomical and physiological differences predispose the pediatric patients to development of respiratory depression or arrest. Continuous patient monitoring and conversation with patient is essential to rule out the state of deep sedation. However, if a patient goes into an unplanned state of deep sedation or unconsciousness, the effects can be catastrophic. Use of intra-operative jaw elevation is an essential component for patient safety and must be employed.

Application of head tilt-chin lift and jaw thrust techniques for an extended time period is physically strenuous for the anaesthetists. Recently some prefabricated devices which are able to elevate the jaw for extended time period are available in the market. There is need for studies in near future to evaluate the efficacy of these devices to maintain the patency of the airway.

\section{References}

[1] Bennett CR (1990) Monheim's Local Anesthesia and Pain Control in Dental Practice. CBS Publication, Delhi, India.

[2] McDonald RE, Avery DR \& Dean JA (2004) Dentistry for the Child and Adolescent. Mosby, St. Louis, Missouri.

[3] Malamed SF (2007) Medical Emergencies in the Dental Office. Mosby, Reed Elsevier India.

[4] Quan L \& Franklin WH (2000) Ventricular Fibrillation: A Pediatric Problem. Futura Publishers, Armonk, New York.

[5] Goodson JM \& Moore PA (1983) Life-threatening reactions after pediatric sedation: an assessment of opioid, local anesthetic, and antiemetic drug interaction. Journal of American Dental Association 107, 239-45.

[6] 2005 International consensus on cardiopulmonary resuscitation (CPR) and emergency cardiovascular care (ECC) science with treatment recommendations. Circulation 112, 1-125.

[7] Kuisma M, Suominen P \& Korpela R (1995) Paediatric out-ofhospital cardiac arrest: epidemiology and outcomes. Resuscitation 30, 141-50.

[8] Sirbaugh PE, Pepe PE, Shook JE, Kimball KT, Goldman MJ, Ward MA \& Mann DM (1999) A prospective, population-based study on the demographic, epidemiology, management, and outcome of out-ofhospital pediatric cardiopulmonary arrest. Annals of Emergency Medicine $33,174-84$.

[9] Schmitt ER \& Gausche-Hill M (2011) Advanced Pediatric Airway Management- Updates and Controversies. Emergency Medicine and Critical Care. 2011; 5:21-7. 